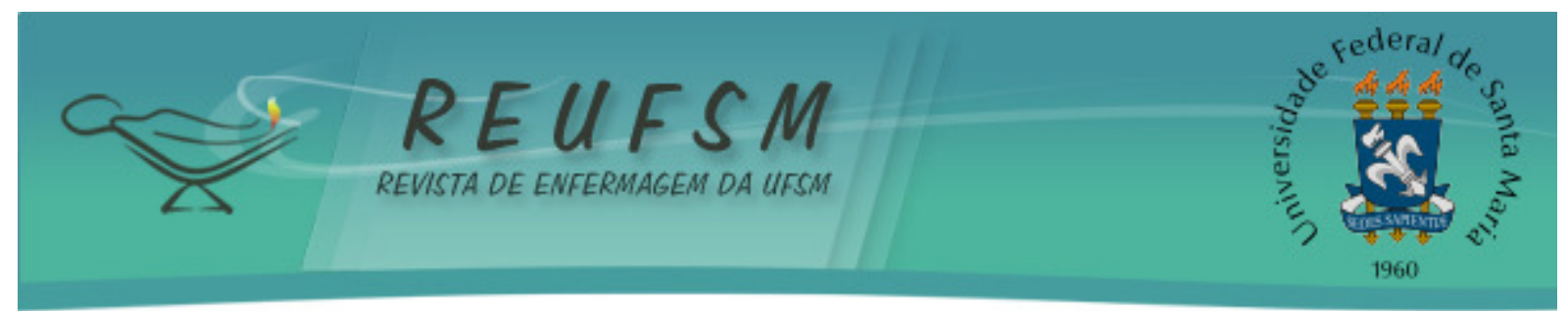

\title{
ARTIGO ORIGINAL AÇÕES DE CUIDADO PARA A PROMOÇÃO DA SEGURANÇA AO PACIENTE EM VENTILAÇÃO MECÂNICA INVASIVA
}

\author{
CARE ACTIONS FOR THE PROMOTION OF SAFETY TO THE PATIENT IN \\ INVASIVE MECHANICAL VENTILATION
}

ACCIONES DE CUIDADO PARA LA PROMOCIÓN DE LA SEGURIDAD AL PACIENTE EN VENTILACIÓN MECÁNICA INVASIVA

\author{
Fabiana Cristina Pires ${ }^{1}$ \\ Julia Maria Vergani Fanan ${ }^{2}$ \\ Juliana da Silva Garcia Nascimento ${ }^{3}$
}

\section{Doi: $10.5902 / 2179769225685$}

RESUMO: Objetivo: verificar o conhecimento da equipe de enfermagem e fisioterapia sobre as ações de cuidado relacionadas ao uso de ventilação mecânica. Método: estudo descritivo, exploratório, quantitativo, realizado na unidade de terapia intensiva com 32 participantes da equipe multiprofissional, no período de setembro a novembro de 2016, por meio de questionário autoexplicativo. Resultados: foram identificadas duas principais vertentes sobre as ações de cuidado relacionadas ao uso de ventilação mecânica invasiva. A primeira abordou quatro estratégias de cuidado direcionadas ao ventilador mecânico e a segunda relacionou quatro cuidados específicos voltados ao paciente critico ventilado mecanicamente. Conclusão: o conhecimento sobre as estratégias de cuidado foi satisfatório quanto às condutas referentes ao ventilador mecânico e ao paciente, valorizando a adoção de protocolos baseados em evidências científicas sobre a temática.

Descritores: Respiração artificial; Cuidados críticos; Unidades de terapia intensiva; Equipe de assistência ao paciente.

ABSTRACT: Aim: to verify the knowledge of the nursing and physiotherapy teams about the care actions related to the use of mechanical ventilation. Method: a descriptive, exploratory, quantitative study performed in the intensive care unit with 32 participants from the multiprofessional team, from September to November, 2016, through a self-explanatory questionnaire. Results: two main aspects of care actions related to the use of invasive mechanical ventilation were identified, the first one addressed four strategies of care directed to the mechanical ventilator and the second one related four specific care directed to the critical patient ventilated mechanically. Conclusion: the knowledge about the care strategies was satisfactory regarding the mechanical ventilator and patient behavior, valuing the adoption of protocols based on scientific evidences on the subject.

Descriptors: Respiration artificial; Critical care; Intensive care units; Patient care team.

\footnotetext{
1 Graduanda em Enfermagem. Universidade de Uberaba, Uberaba, Minas Gerais, Brasil, e-mail: enfermagem.pires@gmail.com

${ }^{2}$ Fisioterapeuta. Universidade de Uberaba, Uberaba, Minas Gerais, Brasil, e-mail:juliafanan@outlook.com

3 Enfermeira. Mestre. Universidade de Uberaba, Uberaba, Minas Gerais, Brasil, e-mail: mestradounesp28@yahoo.com.br
} 


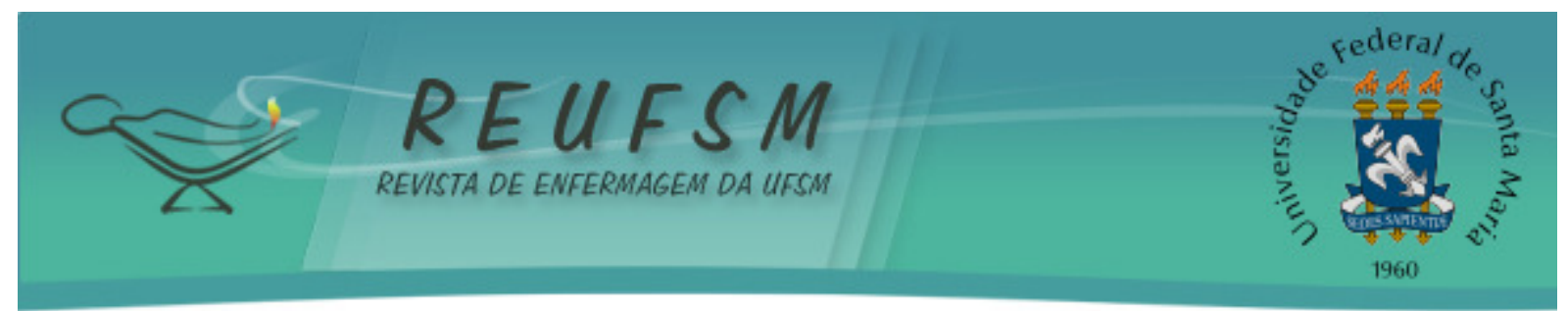

RESUMEN: Objetivo: verificar el conocimiento del equipo de enfermería y fisioterapia sobre las acciones de cuidado relacionadas al uso de ventilación mecánica. Método: estudio descriptivo, exploratorio, cuantitativo, realizado en la unidad de terapia intensiva con 32 participantes del equipo multiprofesional, en el período de septiembre a noviembre de 2016, por medio de un cuestionario autoexplicativo. Resultados: se identificó dos principales vertientes sobre las acciones de cuidado relacionadas al uso de ventilación mecánica invasiva, la primera trató de cuatro estrategias de cuidado dirigidas al ventilador mecánico y la segunda relacionó cuatro cuidados específicos dirigidos al paciente crítico ventilado mecánicamente. Conclusión: el conocimiento sobre las estrategias de cuidado fue satisfactorio en relación a las conductas referentes al ventilador mecánico y al paciente, valorizando la adopción de protocolos basados en evidencias científicas sobre la temática.

Descriptores: Respiración artificial; Criticalcare; Unidades de cuidados intensivos; Grupo de atención al paciente.

\section{INTRODUÇÃO}

O paciente crítico requer cuidados específicos por apresentar alterações orgânicas significativas, o que torna essencial a assistência com profissionais capacitados. ${ }^{1} \mathrm{E}$ dentre as modalidades de cuidado utilizadas em Unidade de Terapia Intensiva (UTI), destaca-se a ventilação mecânica invasiva. ${ }^{1}$

A ventilação mecânica invasiva é uma forma de tratamento ventilatório artificial utilizada para os casos graves ou refratários de insuficiência respiratória aguda. Trata-se de uma terapia utilizada quando o indivíduo não realiza as trocas gasosas de maneira eficaz. ${ }^{2}$

Este modo ventilatório está associado a complicações agravam o estado clínico do paciente, como o barotrauma, volutrauma, comprometimento da função de outros órgãos e infecção. ${ }^{3}$

Frente à possibilidade de tais eventos iatrogênicos, as principais ações de cuidado configuram-se em: controle de sinais vitais, monitorização de trocas gasosas, aspiração de secreções pulmonares, higiene oral, controle da pressão do balonete, umidificação e aquecimento do gás inalado e controle de infecção. ${ }^{4}$

A existência de ventiladores mecânicos cada vez mais tecnológicos motivou a necessidade da discussão por especialistas brasileiros sobre o suporte ventilatório. Houve a análise das evidências científicas sobre o fornecimento de ventilação segura que pudessem subsidiar os profissionais para uma assistência de qualidade. ${ }^{5}$

Reuniu-se, desta maneira, o conhecimento sobre 29 temas referentes à ventilação mecânica invasiva em um documento denominado, Diretrizes Brasileiras de Ventilação Mecânica, cujas informações apresentaram impacto positivo no atendimento ventilatório. ${ }^{5}$ 


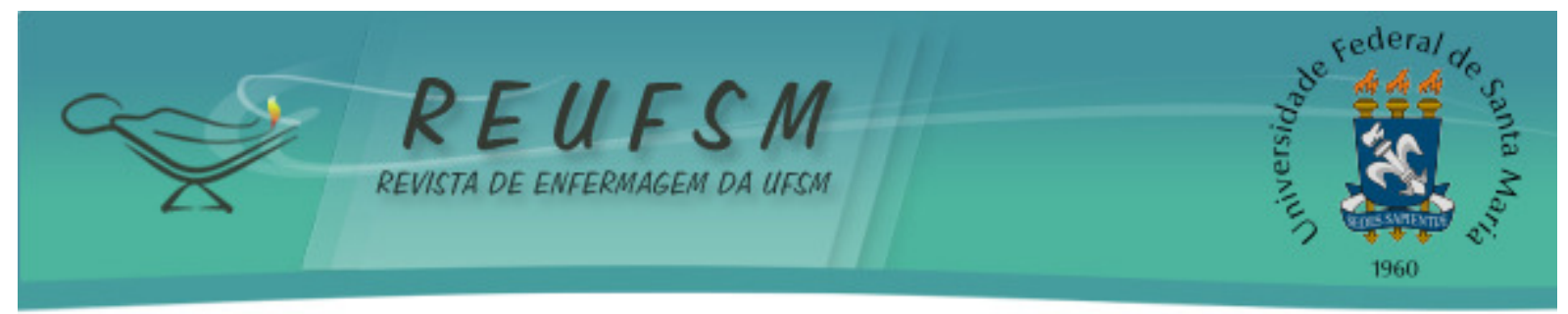

As Diretrizes Brasileiras de Ventilação Mecânica contribuem para uma assistência à saúde isenta de riscos e falhas, além de subsidiarem cientificamente a elaboração de Bundles de prevenção direcionados a Pneumonia Associada à Ventilação Mecânica (PAVM). ${ }^{5-6-7-8}$

As equipes de enfermagem e fisioterapia tem sido o grande diferencial no cuidado ventilatório de doentes críticos, o que requer constante atualização para a manutenção da excelência no cuidado. Porém, estes profissionais, encontram inúmeras questões conflitantes relacionadas a ventilação mecânica, e se tornam atores essenciais para a implementação de estratégias preventivas. ${ }^{9}$

Aplicar bundles de prevenção na prática assistencial para a utilização segura da ventilação invasiva, constitui-se em um desafio, na perspectiva de permitir a avaliação contínua da assistência e a criação de metas terapêuticas claras. ${ }^{6}$

Compreende-se, a necessidade da aquisição de conhecimento pela equipe de enfermagem e fisioterapia, sobre a assistência adequada quanto ao suporte ventilatório invasivo, na intencionalidade de estabelecer segurança frente às intervenções propostas.

Esta necessidade contribui para justificar este estudo quanto ao aprofundamento sobre o tema, na expectativa da elaboração de protocolos que abordem as ações de cuidado para promoção da segurança do paciente ventilado mecanicamente.

Diante deste contexto, questiona-se: o conhecimento da equipe de enfermagem e fisioterapia sobre as ações de cuidado necessárias quanto ao uso de ventilação mecânica invasiva na unidade de terapia intensiva é satisfatório? Desta forma, este estudo tem como objetivo verificar o conhecimento da equipe de enfermagem e fisioterapia sobre as ações de cuidado relacionadas ao uso de ventilação mecânica invasiva.

\section{MÉTODO}

Estudo descritivo, exploratório, de abordagem quantitativa, realizado na UTI adulto de um hospital universitário do interior do Estado de Minas Gerais, composta por 20 leitos, no período de setembro a novembro de 2016. Em relação às equipes envolvidas neste estudo, o setor é composto por 7 enfermeiros, 5 fisioterapeutas e 24 profissionais técnicos em enfermagem.Compuseram o estudo, todos os profissionais da equipe de enfermagem e fisioterapia do setor, exceto 4 técnicos em enfermagem que encontravam-se afastados do trabalho no período da coleta, totalizando 32 profissionais. 


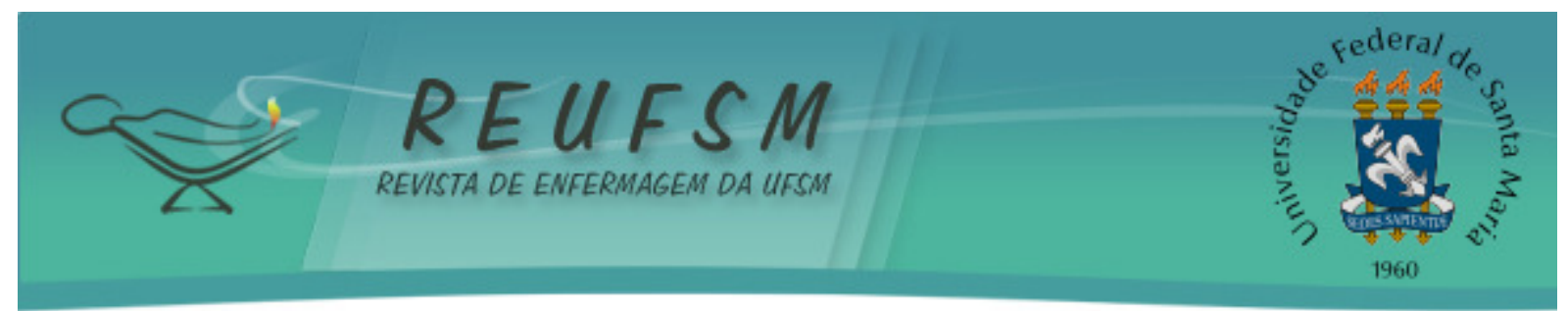

Foram incluídos no estudo colaboradores da equipe de enfermagem e fisioterapia, responsáveis pelo cuidado direto com paciente em ventilação mecânica invasiva. Foram excluídos colaboradores em período de férias ou afastamentos em geral na escala de trabalho e os profissionais médicos, pelo fato do objeto de estudo da pesquisa abordar o conhecimento específico sobre as ações direcionadas à equipe de enfermagem e fisioterapia.

Os dados foram coletados por meio de um questionário autoexplicativo, elaborado pelos pesquisadores, abordando dois domínios: o perfil da amostra, contendo seis questões fechadas relacionadas à categoria profissional, sexo, idade, tempo de formação e atuação na UTI e a presença de especialização. O segundo abordou o conhecimento quanto às estratégias de cuidado, contendo 16 questões fechadas.

Para maior fidedignidade, o instrumento foi avaliado por três especialistas em ventilação mecânica e UTI, e realizado teste piloto com cinco profissionais do setor em questão, para posterior adequação do questionário.

Os achados foram tabulados em planilhas no Programa Microsoft Excel, validados por dupla digitação, exportados e processados no programa Statistical Package for the Social Sciences (SPSS), versão 22 para Windows. As variáveis qualitativas foram analisadas segundo estatística descritiva, por meio de distribuição de frequência absoluta e relativa.

A pesquisa foi conduzida de acordo com os padrões éticos definidos, sendo aprovada pelo Comitê de Ética e Pesquisa com o número de parecer 1.569.577 em $1^{\circ}$ de junho de 2016, cumprindo assim, as exigências da Resolução 466/12 do Conselho Nacional de Saúde. Os participantes do estudo foram consultados quanto ao interesse e à disponibilidade em participar, e assinaram o Termo de Consentimento Livre e Esclarecido.

\section{RESULTADOS}

A maioria dos participantes era do sexo feminino (75\%). A categoria profissional predominante foi a dos técnicos de enfermagem (62,5\%). A faixa etária prevalente foi entre 25 e 30 anos (43,8\%). O tempo de formação dos participantes foi de 6 a 10 anos $(59,4 \%)$, e o tempo de atuação em UTI adulto predominante foi de 5 anos $(68,8 \%)$.O perfil da amostra é apresentado na Tabela 1 a seguir. 


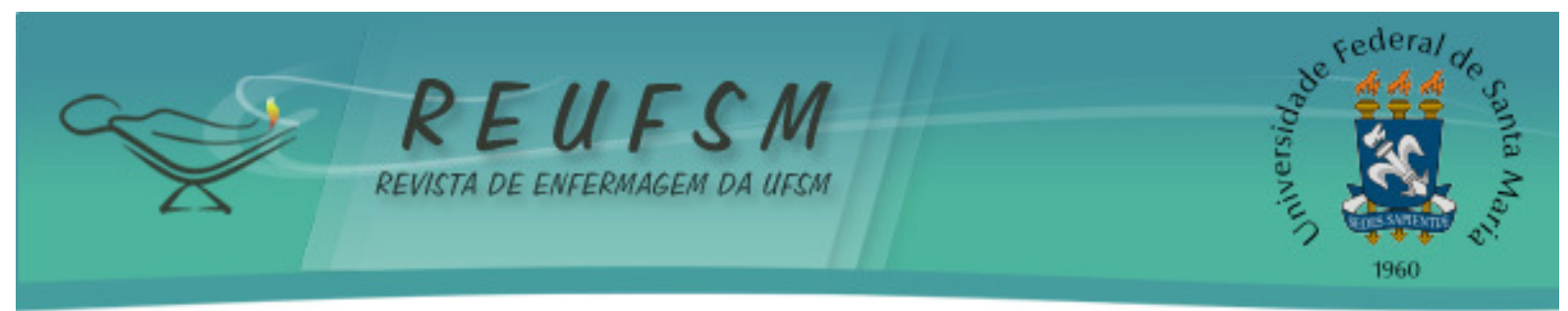

Tabela 1 - Distribuição das variáveis sociodemográficas dos profissionais da unidade de terapia intensiva. Uberaba, 2017. $(\mathrm{N}=32)$

\begin{tabular}{lcc}
\hline Variáveis & N & \% \\
\hline Sexo & 24 & 75 \\
$\quad$ Feminino & 8 & 25 \\
Masculino & & \\
Categoria profissional & 7 & 21,9 \\
$\quad$ Enfermeiro & 20 & 62,5 \\
Técnico de enfermagem & 5 & 15,6 \\
Fisioterapeuta & & \\
Faixa etária, anos & & \\
18-24 anos & 14 & 43,8 \\
$25-30$ anos & 10 & 31,2 \\
$31-40$ anos & 5 & 15,6 \\
Acima de 40 anos & & 34,4 \\
Tempo de formação & 11 & 59,4 \\
Até 5 anos & 19 & 3,1 \\
6 -10 anos & 1 & 3,1 \\
$11-15$ anos & 1 & \\
Acima de 20 anos & & 68,8 \\
Tempo de atuação & 22 & 28,1 \\
Até 5 anos & 9 & 0 \\
6-10 anos & 0 & 3,1 \\
$11-15$ anos & 1 & 0 \\
$16-20$ anos & 0 & \\
Acima de 20 & &
\end{tabular}

A Tabela 2 apresenta o conhecimento sobre o cuidado ideal quanto a conduta tomada em relação à presença de água ou de sujidade no circuito do ventilador mecânico, à frequência de troca dos circuitos na ausência de sujidades, à frequência de troca do filtro do ventilador e o profissional responsável pela troca do filtro. 


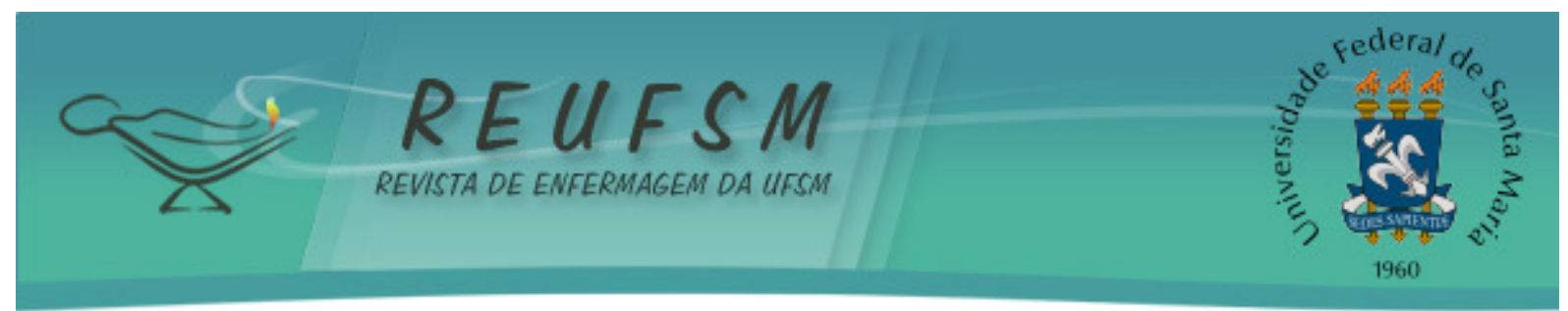

Tabela 2- Ações de cuidado referentes ao ventilador mecânico pela equipe de enfermagem e fisioterapia da unidade de terapia intensiva, Uberaba, 2017. $(\mathrm{N}=32)$

\begin{tabular}{lcc}
\hline Variáveis & N & \% \\
\hline $\begin{array}{l}\text { Conduta a ser tomada quando há água no circuito de ventilador ou } \\
\text { presença de sujidade }\end{array}$ & & \\
$\quad$ Remover água e, em caso de sujidade, aguardar a data da troca do & 1 & 3,1 \\
$\quad$ circuito & 23 & 71,9 \\
$\quad$ Remover água e, em caso de sujidade, realizar a troca do circuito & 7 & 21,9 \\
$\quad$ rrocar todo o circuito & 1 & 1 \\
$\quad$ Apenas remover a água & 0 & 0 \\
$\quad$ Não sei & & \\
Frequência de troca dos circuitos de ventilação mecânica na ausência & & \\
de sujidade & 29 & 90,6 \\
A cada 7 dias & 0 & 0 \\
A cada 10 dias & 1 & 3,1 \\
A cada 15 dias & 0 & 0 \\
A cada 20 dias & 2 & 6,3 \\
Não sei & 2 & 6,3 \\
Frequência de troca do filtro do ventilador mecânico & 1 & 3,1 \\
$\quad$ Todos os dias & 27 & 84,3 \\
1 vez na semana & & 0 \\
Ao menos 1 vez na semana e quando houver sinal de sujidade & 2 & 6,3 \\
A cada plantão de 6 horas & & \\
Não sei & 0 & 0 \\
Profissionais responsáveis pela troca do filtro do ventilador mecânico & 0 & 0 \\
$\quad$ Enfermeiros & 4 & 12,5 \\
Técnicos de enfermagem & 28 & 87,5 \\
Fisioterapeutas & 0 & 0 \\
$\quad$ Toda a equipe de enfermagem e de fisioterapia & & \\
Não sei &
\end{tabular}

Se houvesse água ou sujidade no circuito, 71,9\% assinalaram como correta a conduta de remover a água e, em caso de sujidade, realizar a troca do circuito. Sobre a frequência de troca dos circuitos do ventilador mecânico na ausência de sujidade, 90,6\% optaram pela troca a cada 7 dias. Para 84,3\%, a resposta correta foi a de ao menos uma vez na semana ou quando houver sujidade. Para $87,5 \%$ dos participantes, os profissionais responsáveis pela troca do filtro/umidificador e circuito do ventilador mecânico eram as equipes de enfermagem e de fisioterapia.

A Tabela 3 evidencia o conhecimento sobre as ações de cuidado direcionadas quanto a higiene oral, posicionamento da cabeceira do leito do paciente e mudança de decúbito. 


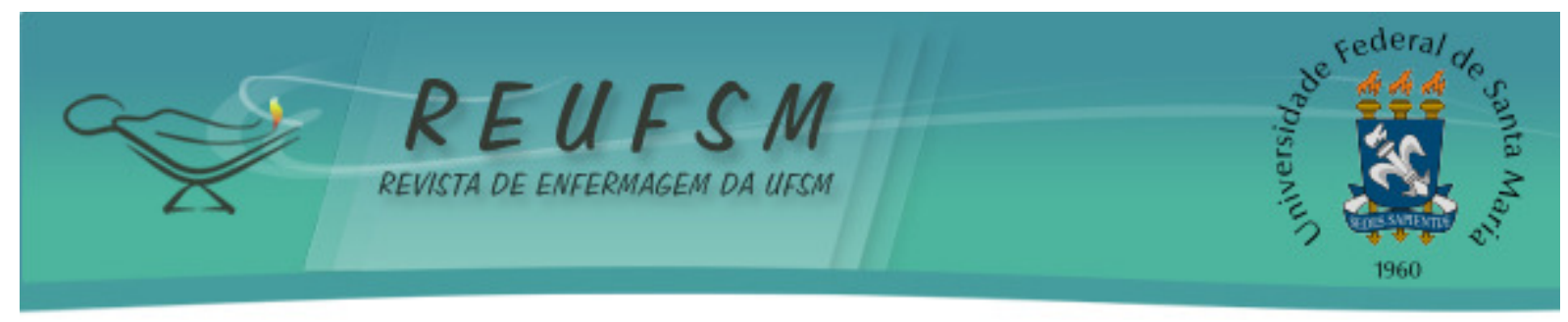

Tabela 3 - Conhecimento das ações de cuidado direcionadas ao paciente em uso de ventilação mecânica invasiva, Uberaba, 2017. ( $\mathrm{N}=32)$

\begin{tabular}{lcc}
\hline Variáveis & N & \% \\
\hline Frequência a ser realizada a higiene oral do paciente, vezes/dia & & \\
1 vez & 3 & 9,4 \\
2 vezes & 4 & 12,5 \\
1 a cada plantão de 6 horas & 24 & 75 \\
A cada 12 horas & 1 & 3,1 \\
Não sei & 0 & 0 \\
Posicionamento ideal da cabeceira do leito do paciente & & \\
Sempre abaixada & 0 & 0 \\
30-45 apenas durante a aspiração & 3 & 9,4 \\
$30-45^{\circ}$ & 28 & 87,5 \\
Acima de 45 & 1 & 3,1 \\
Não sei & 0 & 0 \\
Frequência da mudança de decúbito & & \\
A cada 1 hora & 0 & 0 \\
Após cada banho & 0 & 0 \\
A cada 2 horas & 32 & 100 \\
Uma vez ao dia & 0 & 0 \\
Não sei & 0 & 0 \\
Profissionais responsáveis pela mudança de decúbito & & \\
Enfermeiros & 0 & 0 \\
Enfermeiros e técnicos de enfermagem & 4 & 12,5 \\
Fisioterapeutas & 28 & 87,5 \\
Equipe de enfermagem e fisioterapia & 0 & 0 \\
Não sei & 0 & 0 \\
\hline
\end{tabular}

A resposta predominante para a frequência da higiene oral foi a de uma vez a cada plantão de 6 horas com $75 \%$ das respostas. Sobre o posicionamento ideal da cabeceira do leito, $87,5 \%$ dos participantes indicaram como correta a inclinação de 30 a $45^{\circ}$. Quanto à frequência de mudança de decúbito, unanimemente a resposta foi realizar a mudança de decúbito do paciente a cada 2 horas. Para 87,5\% dos respondentes, os profissionais responsáveis pela mudança de decúbito é a equipe de fisioterapia.

\section{DISCUSSÃO}

Quanto ao perfil dos participantes, houve destaque quanto ao sexo feminino, realidade que corrobora com a literatura, ao apontar a mulher como maioria na prestação dos cuidados no ambiente hospitalar, aspecto que reflete a tradição cultural do cuidado. ${ }^{10}$

A categoria profissional predominante neste estudo foi a dos técnicos em enfermagem, o que também foi evidenciado em um estudo sociodemográfico realizado pelo Conselho Federal de Enfermagem em 2015 que mapeou e caracterizou a enfermagem no Brasil, revelando $77 \%$ de técnicos e auxiliares de enfermagem. ${ }^{11}$ 


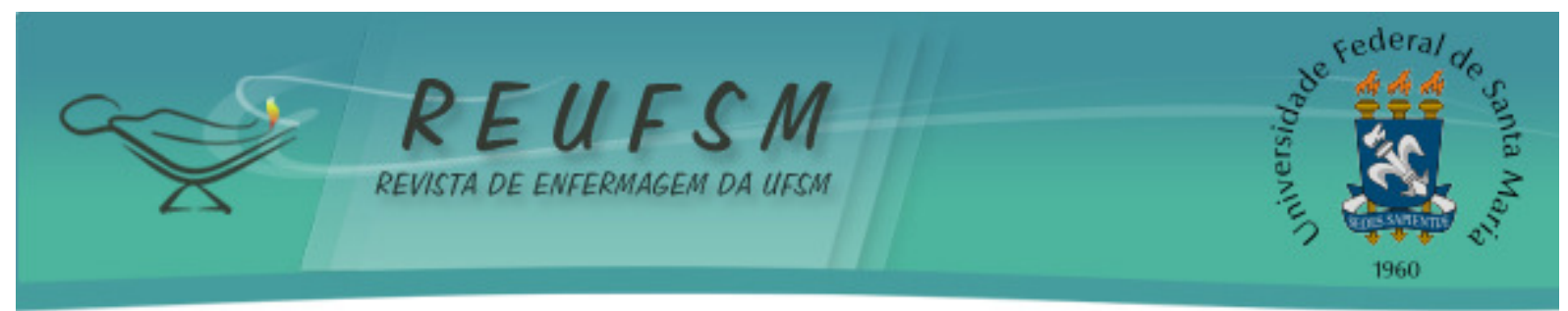

A faixa etária prevalente neste estudo, revelou profissionais jovens na unidade de terapia intensiva, com no mínimo 6 anos de formação e experiência em UTI de 5 anos.

É fato que, a enfermagem é uma profissão em pleno rejuvenescimento no país. Atualmente, registra-se $40 \%$ do contingente da profissão com idade entre 36 e 50 anos; $38 \%$ dos profissionais entre 26 e 35 anos e $2 \%$ apenas com idade acima de 61 anos. ${ }^{11}$

Já, quanto ao tempo de formação e experiência, os artigos relatam experiência da equipe de enfermagem e fisioterapia de, no mínimo, quatro anos em UTI, corroborando com este estudo e evidenciando a vivência dos colaboradores em setores críticos. ${ }^{10}$

O conhecimento das ações de cuidado ao paciente em ventilação mecânica invasiva, identificado pela equipe de enfermagem e fisioterapia da UTI abordou aspectos quanto ao ventilador mecânico e ao paciente.

Quanto à conduta a ser tomada quando houver sinal de água ou sujidade no circuito do ventilador mecânico, a opção maioritária escolhida, neste estudo, foi a de remover a água e, em caso de sujidade, realizar a troca do circuito. Esta afirmação corrobora com a literatura, que indica a troca de circuitos do ventilador somente na presença de sujidade ou danificação, não havendo necessidade de troca periódica. ${ }^{5}$

Percebe-se a que não há um período padrão que estabeleça a rotina de substituição dos circuitos dos ventiladores nos hospitais. Não é apontado pela literatura um tempo exato para esta troca, apenas que, não se deve manipular os circuitos antes de 48 horas se estiverem limpos ${ }^{5-6-12}$.

Um ponto a se destacar quanto ao conhecimento dos participantes, em relação à frequência de troca dos circuitos, foi a indicação do período de troca de 7 dias, diante da ausência de sujidade.

Esta dicotomia sobre a frequência ideal de troca dos circuitos dos ventiladores mecânicos quando limpos, e o padrão de troca destacado no presente estudo, aponta a importância da discussão desta prática, almejando a redução dos custos com o processamento dos artigos ventilatórios, que não apresentam impacto significativo na diminuição das taxas de infecção. ${ }^{13}$

Neste estudo, predominou a frequência de troca do filtro umidificador do ventilador mecânico de, ao menos, uma vez na semana e quando houver sinal de sujidade. A literatura também recomenda a mesma frequência de troca do filtro, em caso de sujidade, condensação ou danos, ${ }^{5}$ corroborando com o conhecimento identificado neste estudo. 


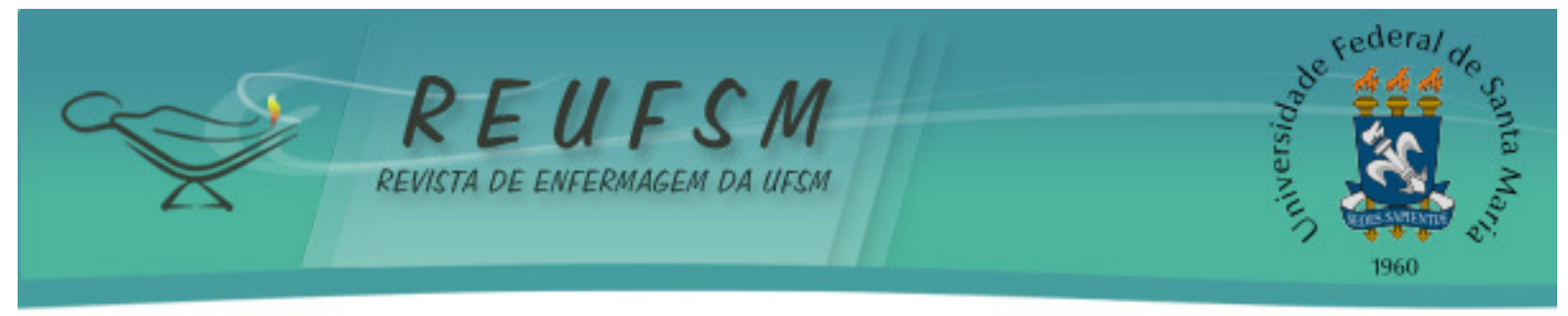

$\mathrm{O}$ uso do filtro umidificador previne a colonização por microrganismos e desenvolvimento da pneumonia associada a ventilação mecânica, funcionando como barreira à entrada de gentes patológicos ou microrganismos. ${ }^{14}$

Diferentemente dos circuitos dos ventiladores, evidencia-se que a troca do filtro deve ser realizada a cada sete dias ou quando necessário, para prevenção da pneumonia associada à ventilação mecânica, já que este funciona como barreira para infecções. ${ }^{14}$

A respeito dos profissionais responsáveis por realizar a troca do filtro umidificador e dos circuitos, a maioria dos participantes apontaram que, ambas as equipes de enfermagem e fisioterapia, são responsáveis por desempenharem tal função. Esta estratégia de cuidado foi proposta como função de ambas as equipes pelo III Consenso Brasileiro de Ventilação Mecânica. ${ }^{5}$

A resposta sobre o profissional responsável pela troca de filtro e circuito do ventilador foi correta neste estudo, portanto, embasando-se no III Consenso Brasileiro de Ventilação Mecânica, que aponta a necessidade do setor em estabelecer a sua rotina quanto ao profissional responsável por esta troca, ou dividi-la entre os profissionais destas equipes. ${ }^{5}$

Quanto aos cuidados referentes ao paciente, evidencia-se que, a higiene oral, realizada por meio de técnicas combinadas de higienização, favorece a redução de focos primários de infecção na boca, e consequentemente o evento da pneumonia associada à ventilação mecânica. ${ }^{15}$

Independente da técnica realizada para higienização oral dos pacientes, é essencial que a equipe de enfermagem esteja preparada para executar este cuidado, garantindo que ela seja realizada de forma segura e rotineira. ${ }^{16}$

No presente estudo, a frequência ideal de realização da higiene oral foi de uma vez a cada plantão de 6 horas, totalizando quatro vezes ao dia, condizendo com a literatura, que reforça a associação entre a pneumonia associada à ventilação mecânica e a não higienização ou higienização precária da cavidade bucal. ${ }^{15}$

Quanto ao cuidado referente ao posicionamento da cabeceira do leito, foi apontada como ideal, nesta pesquisa, a angulação de 30 a 45 graus. Atualmente, recomenda-se que a cabeceira do leito do paciente em ventilação mecânica esteja inclinada de 30 a $45^{\circ}$ devido ao risco de broncoaspiração ${ }^{5}$, além de ser uma posição que permite melhor mecânica respiratória. ${ }^{17}$

A elevação da cabeceira entre $30^{\circ}-45^{\circ}$ é uma das principais recomendações para evitar a broncoaspiração, principalmente nos pacientes que estiverem recebendo nutrição enteral, garantindo eficiente barreira para o desenvolvimento de infecção pulmonar em pacientes críticos. ${ }^{18}$ 


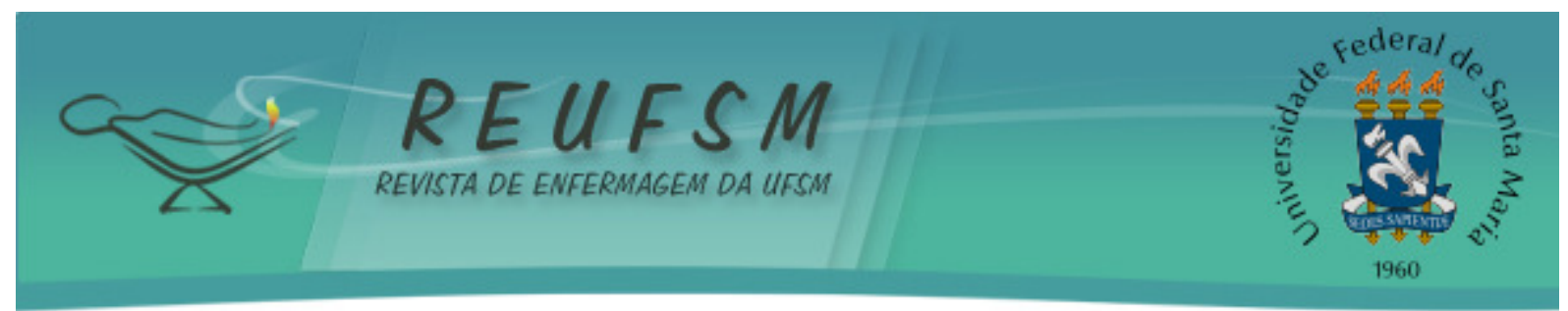

Outro cuidado essencial com o paciente crítico ventilado mecanicamente é a mudança de decúbito, pois movimenta o paciente no leito repercutindo na qualidade das trocas gasosas. ${ }^{19}$

Neste estudo, foi identificado que a mudança de decúbito dos pacientes deve ser realizada a cada 2 horas, o que está de acordo com as Recomendações Brasileiras de Ventilação Mecânica. ${ }^{5}$

Sabendo-se da importância da realização da mudança de decúbito, foi questionado, no presente estudo, quais profissionais eram responsáveis por tal cuidado. A equipe de fisioterapia foi citada como responsáveis pelos participantes.

A literatura recomenda que este cuidado seja realizado em conjunto, por fisioterapeutas e equipe de enfermagem, porém responsabilizando o enfermeiro em grande parte, por esta assistência, por ser o profissional que geralmente elabora os protocolos de prevenção para lesões por pressão. ${ }^{20-21}$

Para desenvolver melhores estratégias de segurança para pacientes críticos em relação ao uso da ventilação mecânica invasiva, é considerada a adoção de bundlesassistenciais que subsidiem o cuidado prestado, tornando-se ferramentas úteis que garantem resultados satisfatórios a clientela. ${ }^{22}$ Incentiva-se assim, a articulação do trabalho do profissional enfermeiro e fisioterapeuta na perspectiva das ações de gestão do cuidado e elaboração de protocolos, em favor da sensibilização da unidade de terapia intensiva quanto aos pacientes ventilados mecanicamente. ${ }^{22}$

\section{CONCLUSÃO}

Foi identificado o conhecimento sobre as ações de cuidado para a promoção da segurança do paciente em ventilação mecânica invasiva, abordando características quanto ao ventilador mecânico e paciente.

$\mathrm{O}$ conhecimento das equipes de enfermagem e fisioterapia da unidade de terapia intensiva demonstrou-se satisfatório, em ambas as estratégias de cuidado pesquisadas, revelando incipiência apenas quanto à frequência de troca dos circuitos do ventilador.

Não se identificaram lacunas quanto à assistência voltada diretamente ao paciente, o que indica a qualidade do cuidado prestado.

Este estudo apresenta limitações quanto ao tamanho da amostra sendo realizado apenas em uma unidade de terapia intensiva, o que restringe a generalização dos resultados. Aponta-se também que foram abordados, apenas os principais cuidados quanto ao paciente ventilado 


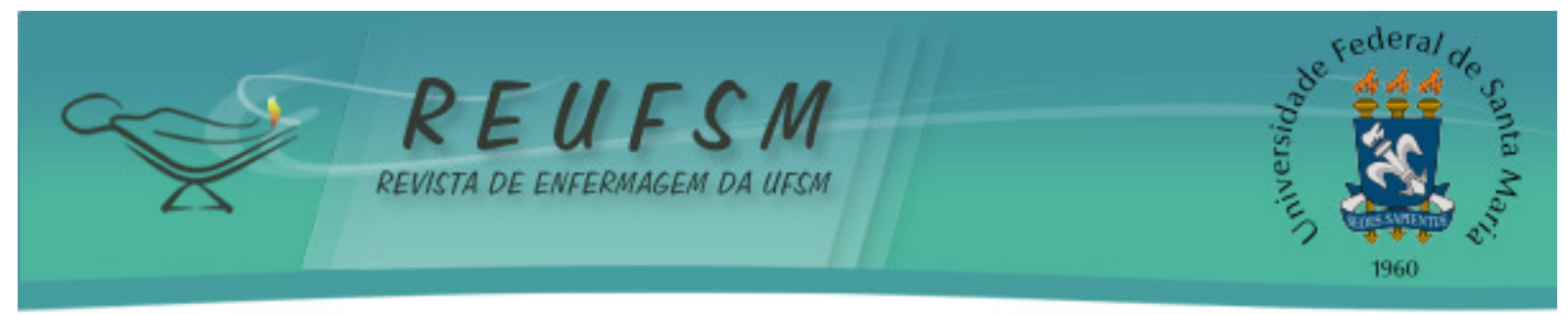

mecanicamente, o que limitou a identificação do conhecimento, porém, baseados em rigor metodológico, e condução da pesquisa, houve a garantia da confiabilidade dos achados.

A pesquisa evidenciou a importância do alinhamento de conhecimento e integração de estratégias da equipe de enfermagem e fisioterapia, por meio da adoção de protocolos baseados em evidências científicas. Sugerem-se, portanto, novas pesquisas, a fim de investigar este conhecimento e esta prática para a promoção da segurança desta clientela.

\section{REFERÊNCIAS}

1. Melo EM, Santos AM, Silveira FM, Sombra RL, Alves RA, Lima VF. Clinical and demographic characteristics of patients on mechanical ventilation in the intensive care unit. Rev Enferm UFPI [Internet]. 2015 [acesso em 2017 jan 29];4(3). Disponível em: http://www.ojs.ufpi.br/index.php/reufpi/article/view/3599.

2. Silva MC, Moura RC. Cuidados de enfermagem na prevenção da pneumonia associada à ventilação mecânica: revisão integrativa. Carpe Diem: Rev Cult Cient UNIFACEX [Internet]. 2016 [acesso em 2017 jan 29];14(2):74-85. Disponível em: https://periodicos.unifacex.com.br/Revista/article/view/854/pdf.

3. Melo EM, Barbosa AA, Silva JLA, Sombra RLS, Studart RMB, Lima FET, et al. Evolução clínica dos pacientes em uso de ventilação mecânica em unidade de terapia intensiva. Rev Enferm UEPE [Internet]. 2015 [acesso em 2017 jun 01];9(2):610-6. Disponível em: https://periodicos.ufpe.br/revistas/revistaenfermagem/article/viewFile/10379/11124.

4. Raurell-Torredà M, Argilaga-Molero E, Colomer-Plana M, Ruiz-García T, Galvany-Ferrer A, González-Pujol A. Intensive care unit profesionals's knowledge about non invasive ventilation comparative analysis. Enferm Intensiva [Internet]. 2015 [acesso em 2017 jun 01];26(2):46-53. Disponível em: https://www.ncbi.nlm.nih.gov/pubmed/25841590.

5. Barbas CSV, Isola AM, Farias AMC, Cavalcanti AB, Gama AMC, Duarte ACM, et al. Recomendações brasileiras de ventilação 2013 - Parte I. Rev Bras Ter Intensiva [Internet]. 2014 [acesso em 2017 jun 01];26(2):89-121. Disponível em: http://www.scielo.br/pdf/rbti/v26n2/0103-507X-rbti-26-02-0089.pdf.

6. Barbosa TP, Oliveira GAA, Lopes MNA, Poletti NAA, Beccaria LM. Praticas assistenciais para segurança do paciente em unidade de terapia intensiva. Acta Paul Enferm [Internet]. 2014 [acesso em 2017 jun 01];27(3):237-42. Disponível em: http://www2.unifesp.br/acta/pdf/v27/n3/v27n3a0.pdf\#page=57.

7. Dal Sasso GTM, Barra DC, Paese F, Almeida SR, Rios GC, Marinho MM, Debétio MG. Processo de enfermagem informatizado: metodologia para associação da avaliação clínica, diagnósticos, intervenções e resultados. Rev Esc Enferm USP [Internet]. 2013 [acesso em 2017 jun 01];47(1):242-9. Disponível em: http://www.scielo.br/scielo.php?script=sci_arttext\&pid=S0080-62342013000100031.

8. Silva SG, Nascimento ERP, Salles RK. Ventilator-associated pneumonia: discourse of professionals about prevention. Esc Anna Nery [Internet]. 2014 [acesso em 2017 jun 


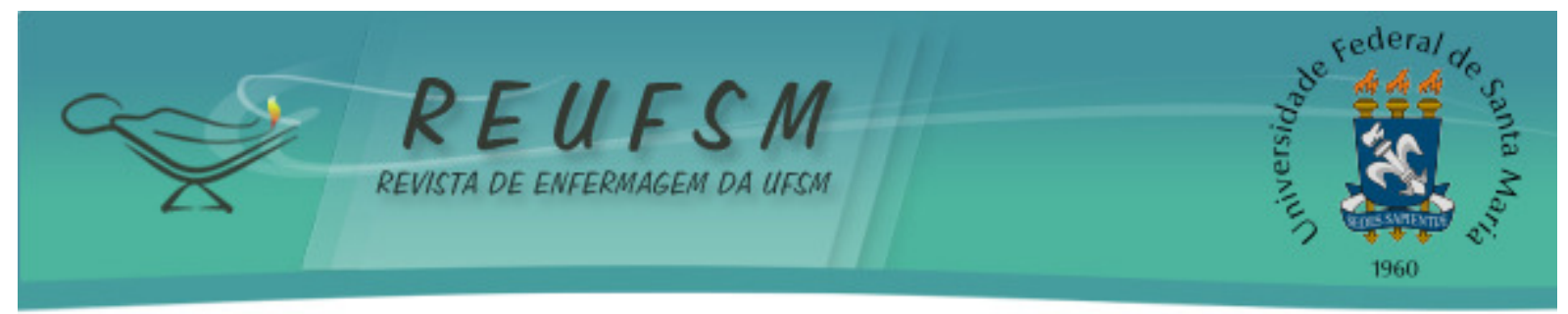

01];18(2):290-5. Disponível em: http://www.redalyc.org/pdf/1277/127730686016.pdf.

9. Mont'alverne DG, Medeiros AI, Silva AG, Carvalho EM. Características dos pacientes sob assistência fisioterapêutica na UTI de um hospital universitário: estudo epidemiológico transversal. Rev Fisioter S Fun [Internet]. 2015 [acesso em 2017 jan 29];5(1):50-8. Disponível

em:

http://repositorio.ufc.br/bitstream/riufc/19345/1/2016_art_\%20dgbmontalverne.pdf.

10. Santos FC, Camelo SH. O enfermeiro que atua em Unidade de Terapia Intensiva: perfil e capacitação profissional. Cultura de los Cuidados [Internet]. 2015 [acesso em 2017 jan 29];19(43):127-40. Disponível em: https://rua.ua.es/dspace/bitstream/10045/52599/1/Cult_Cuid_43_13.pdf.

11. Machado MH, Aguiar Filho W, Lacerda WF, Oliveira E, Lemos W, Wermelinger M, et al. Características gerais da enfermagem: o perfil sócio demográfico. Enferm Foco [Internet]. 2015 [acesso em 2017 jan 29];6(1/4):11-7. Disponível em: http://revista.portalcofen.gov.br/index.php/enfermagem/article/viewFile/686/296.

12. Gonçalves EO, Lima MS, Melo JL, Pontes MSR, Sousa AOB, Albernaz MP. Práticas assistenciais de enfermagem e prevenção da pneumonia associada à ventilação mecânica em UTI. Rev Enferm UFPE [Internet]. 2015 [acesso em 2017 jul 13];9(12):1069-77. Disponível em: https://periodicos.ufpe.br/revistas/revistaenfermagem/article/view/10809/11984.

13. Santiago-Arana C, Zalazar-Cerafino MC. Reuso y sustitucióndel material y equipodesechable de inhaloterapia. Rev Mexicana de Enfermeira Cardiológica [Internet]. 2014 [acesso em 2017 jan 29];22(1):30-2. Disponível em: http://www.medigraphic.com/pdfs/enfe/en-2014/en141f.pdf.

14. Craveiro CM, Cruz IC. Conscience and mechanical ventilation: sistematic literature review for a clinical protocol. J Specialized Nursing Care [Internet]. 2016 [acesso em 2017 jan 29];8(1). Disponível em: http://www.uff.br/jsncare/index.php/jsncare/article/view/2849/700.

15. Saldanha KFD, Costa DC, Pinto SF, Gartti Jardim EC. Avaliação do índice de higiene do paciente crítico. Arch Health Invest [Internet]. 2015 [acesso em 2017 jan 29];4(6):47-53. Disponível em: http://www.archhealthinvestigation.com.br/ArcHI/article/viewFile/1290/1572.

16. Wakiuchi J, Fontes MC, Papa MA. Oral hygiene in patients under mechanical ventilation: integrative review. Rev Enferm UFPE. 2014;8(7):2479-86.

17. Mansano FPN, Belei RA, Vinci LAS, Melo BLD, Cardoso LTQ, Garcia JCP. Impacto de ação educativa na manutenção do decúbito elevado como medida preventiva de pneumonia associada à ventilação mecânica em Unidade de Terapia Intensiva. ABCS Health Sci [Internet]. 2017 [acesso em 2017 jun 01];42(1):21-6. Disponível em: https://nepas.emnuvens.com.br/abcshs/article/viewFile/945/757.

18. Martinez DR, Silva VS, Nepomuceno Jr. BR, Alves GA, Gomes Neto M, Forgiarini Jr. LA. Influência de diferentes graus de elevação da cabeceira na mecânica respiratória de pacientes ventilados mecanicamente. Rev Bras Ter Intensiva [Internet]. 2015 [acesso em 2017 jan 29];27(4):347-52. Disponível em: http://www.scielo.br/scielo.php?pid=S0103507X2015000400347\&script=sci_abstract\&tlng=pt.

19. Hinkle JL, Cheever KH. Brunner \& Suddarth - Tratado de enfermagem médico-cirúrgica. 


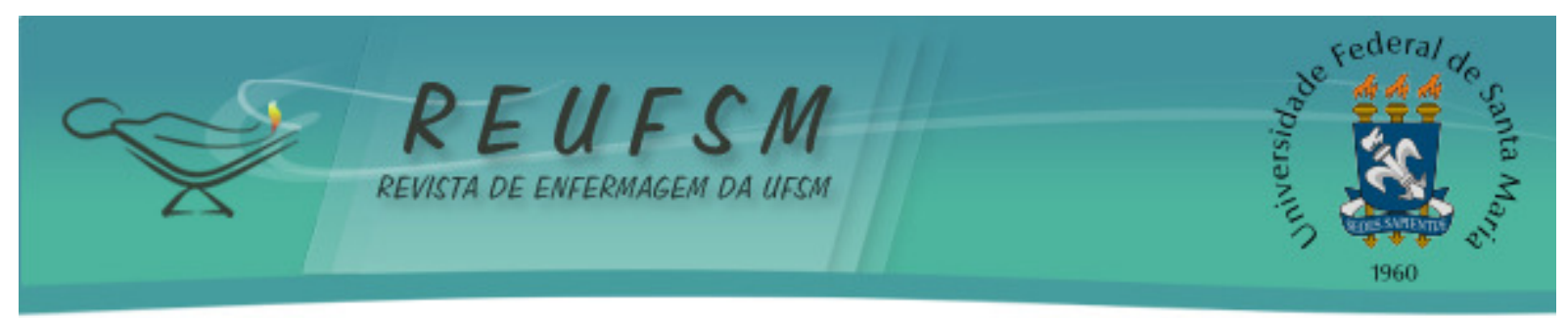

$13^{\mathrm{a}}$ ed. Rio de Janeiro-RJ: Guanabara Koogan; 2016.

20. Agência Nacional de Vigilância Sanitária (Anvisa). Medidas de prevenção de infecção relacionadas à assistência à saúde. 2013 [acesso em 2017 jan 29]. Disponível em http://www20.anvisa.gov.br/segurancadopaciente/images/documentos/livros/Livro4MedidasPreven caoIRASaude.pdf.

21. Moraes JT, Borges EL, Lisboa CR, Cordeiro DC, Rosa EG, Rocha NA. Conceito e classificação de lesão por pressão: atualização do National Pressure Ulcer Advisory Panel. R Enferm Cent O Min. 2016;6(2):2292-306.

22. Siman AG, Brito MJM. Mudanças na prática de enfermagem para melhorar a segurança do paciente. Rev Gaúcha Enferm [Internet]. 2016 [acesso em 2017 jan 29];37(Esp). Disponível em: http://www.scielo.br/pdf/rgenf/v37nspe/0102-6933-rgenf-1983-14472016esp68271.pdf.

Data de submissão: 01/02/2017

Data de aceite: 24/08/2017

Autor principal: Júlia Maria Vergani Fanan

Endereço postal: Rua da Constituição, 1180, Uberaba, Minas Gerais.

Email: juliafanan@outlook.com 